\title{
Persistent Trigeminal Artery: An Unusual Rescue Vessel Collateralization?
}

\author{
Piergiorgio Lochner, Giulio Zuccoli, Francesco Brigo, Frediano Tezzon, Eugen Trinka, \\ Raffaele Nardone
}

Can J Neurol Sci. 2013; 40: 429-430

The persistent trigeminal artery (PPTA) is one of the most common anomalies of the carotid-basilar anastomoses. Persistent trigeminal artery variants originate directly from the precavernous portion of the internal carotid artery (ICA) and may be observed, usually as an incidental finding, in $0.1-1 \%$ of angiography or non-invasive vascular imaging studies. ${ }^{1}$

Little is known about the significance of the PPTA in occlusive cerebrovascular disease.

We describe a patient with PPTA associated with homolateral hypoplasia of the anterior cerebral artery (ACA) and occlusion of the ICA. A protective hemodynamic role of PPTA in this patient is hypothesized and briefly discussed.

The 81-year-old man was admitted because of a severe occipital headache. His past medical history was notable only for arterial hypertension. A lumbar puncture ruled out the presence of subarachnoidal blood. Cerebral magnetic resonance imaging did not reveal areas of restricted diffusion, whereas magnetic resonance angiography showed a right PPTA associated with a hypoplastic precommunicating part of the homolateral ACA (A1) and an occlusion of the right ICA (Figure 1). The Saltzman type 1 PPTA anastomesed with the parasellar right ICA providing vascular supply to the right middle cerebral artery (MCA).

An echocontrast-enhanced ultrasound of the extracranial arteries showed a proximal occlusion of the right ICA. The transcranial-color-coded duplex sonography (TCCD) showed a normal anterograde flow in the right MCA and in the left ophthalmic artery (OA), but a retrograde flow in the right OA. Transesophageal echocardiography and Holter electrocardiogram were unremarkable. The headache can thus be attributed to a distension of collateral arteries in the circle of Willis (the socalled Willis headache). A prophylaxis with acetylsalicylic acid was started.

The relationship between PPTA and occlusive cerebrovascular disease is unclear. It has been suggested that the presence of PPTA may not significantly alter the occurrence of neurological abnormalities in patients with occlusive cerebrovascular insufficiency. ${ }^{2}$ On the other hand, reversal of flow through the PPTA has been demonstrated in patients with severe ICA stenosis. ${ }^{3}$ A collateral support via a PPTA has been also recognized in five patients who underwent the balloon occlusion test of the ICA. ${ }^{4}$

Since we demonstrated a retrograde flow in the right $\mathrm{OA}$, in our patient the right ICA and its middle cerebral branches are with high probability perfused by retrograde flow through the PPTA. Unfortunately, conventional cerebral angiography could

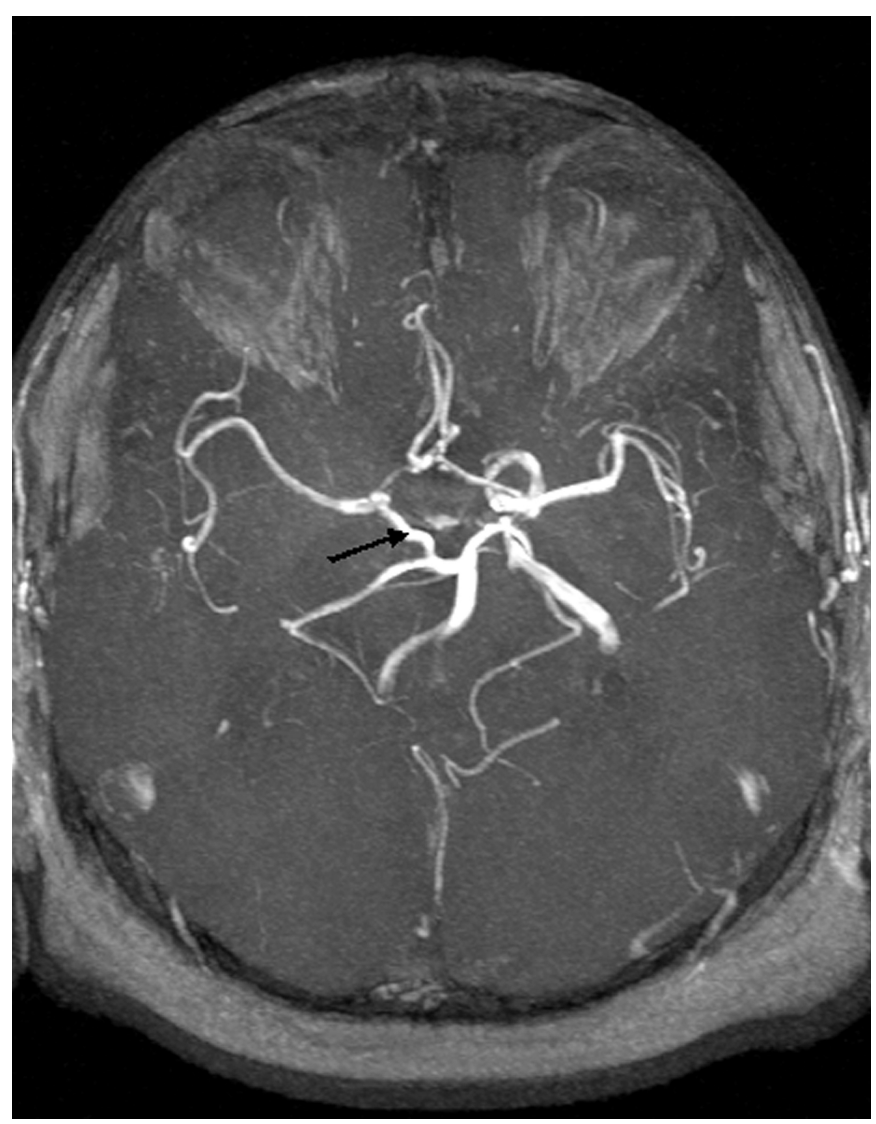

Figure 1: Magnetic resonance angiography: Time of flight showed a persistent medially coursing trigeminal artery (arrow) connecting the right internal carotid artery communicating segment $(C 7)$ and the basilar artery. The overview of the circle of Willis shows this anomaly and an hypoplastic right Al segment.

From the Department of Neurology (PL, FB, FT, RN), Franz Tappeiner Hospital, Meran/o; Department of Neurological, Neuropsychological, Morphological and Movement Sciences (FB), Section of Clinical Neurology, University of Verona, Verona, Italy; Department of Pediatric Radiology (GZ), Children's Hospital Pittsburgh, Pittsburgh, PA, USA; Department of Neurology (ET, RN), Christian Doppler Clinic, Paracelsus Medical University, Salzburg, Austria.

Received October 5, 2012. Final Revisions Submitted December 11, 2012. Correspondence to: Piergiorgio Lochner, Department of Neurology, Franz Tappeiner Hospital, Meran/o, Italy, Via Rossini, 5, 39012, Italy.

Email: piergiorgio.lochner@googlemail.com. 


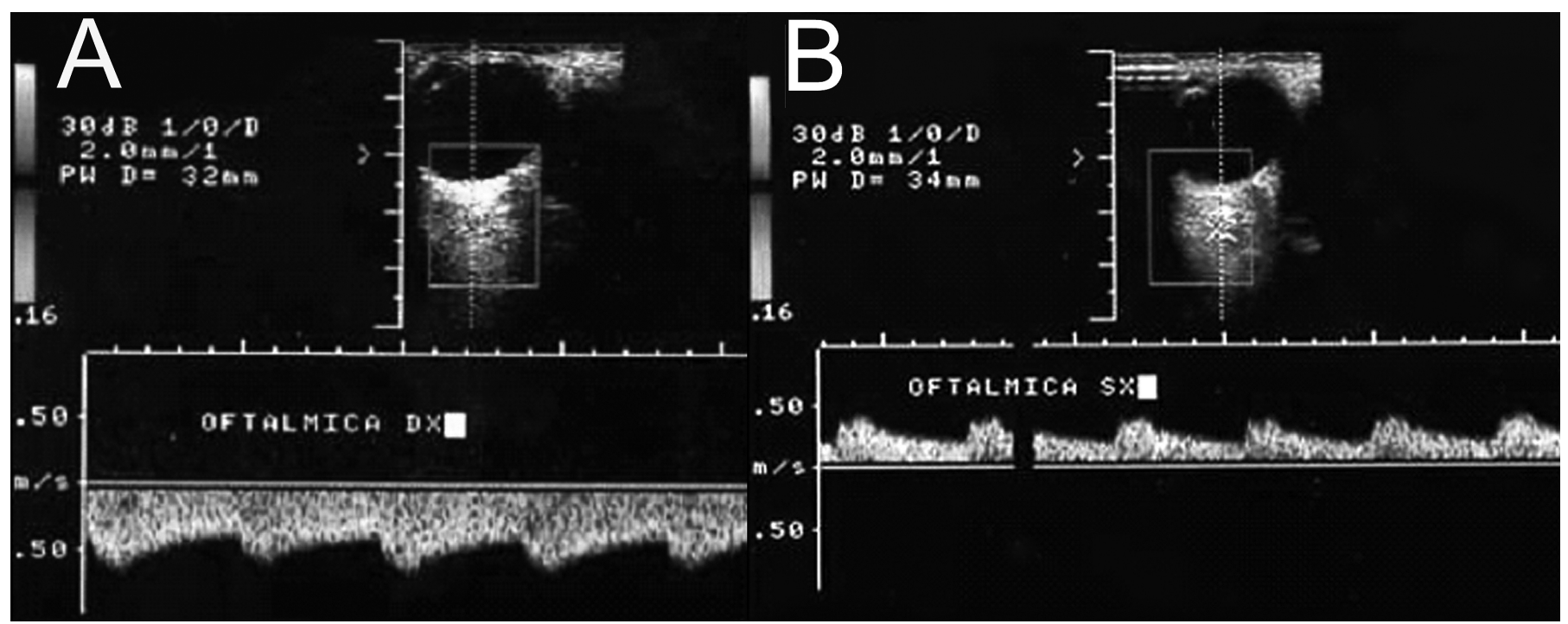

Figure 2: Transcranial-color-coded duplex sonography via the transorbital approach showed a retrograde flow in the right ophthalmic artery (Panel A) and a normal anterograde flow in the left ophthalmic artery (Panel B).

not be performed due to slight renal failure and we were not able to clearly visualize a retrograde flow through the PPTA because of poor insonation conditions. However, it can be assumed that a retrograde flow also occurred in all cerebral arteries which may serve as collaterals, including the PPTA.

Therefore, we conclude that, despite the complete occlusion of the proximal ACI, the presence of the PPTA contributed to keep the patient free of symptoms.

Our case report suggests that PPTA may have a protective hemodynamic role in the presence of an acquired occlusion of homolateral ICA, similar to that reported in patients with congenital absence of the ICA., ${ }^{5,6}$ Therefore, the assessment of the circle of Willis may provide useful information in the clinical setting of occlusive carotid artery disease.

\section{REFERENCES}

1. Suttner N, Mura J, Tedeschi H, et al. Persistent trigeminal artery: a unique anatomic specimen-analysis and therapeutic implications. Neurosurgery. 2000;47(2):428-33.

2. Khodadad G. Trigeminal artery and occlusive cerebrovascular disease. Stroke. 1977;8(2):177-81

3. Nielsen $\mathrm{PB}$, Jonson $\mathrm{M}$. Persistent primitive trigeminal artery demonstrated by vertebral arteriography. Am J Roentgenol Radium Ter Nucl Med. 1967;101(1):47-51.

4. Allen JW, Anthony JDA, Nelson PK. Proximal intracranial internal carotid artery branches: prevalence and importance for balloon occlusion test. J Neurosurgery. 2005;102(1):45-52.

5. Hattori T, Kobayashi H, Inoue S, Sakai N. Persistent primitive trigeminal artery associated with absence of internal carotid artery. Surg Neurol. 1998;50(4):352-55.

6. Given II CA, Huang-Helinger F, Baker MD, Chepuri NB, Morris PP. Congenital absence of the internal carotid artery: case reports and review of the collateral circulation. AJNR Am J Neuroradiol. 2001;22(10):1953-59. 